\title{
Impact of quality of reviews on the perceived diagnosticity, purchase intention and the mediating role of perceived EWOM influence in case of B\&B industry
}

\author{
S. Sood* \\ Amity University, Gurgaon, Haryana, India
}

DOI: http://doi.org/10.52814/PJMA.2021.1103

ARTICLE TYPE: Research paper

ARTICLE HISTORY: Submitted: January 2021, Revisions: March 2021, Accepted: March 2021

HOW TO CITE: Sood, S. (2021). Impact of quality of reviews on the perceived diagnosticity, purchase intention and the mediating role of perceived EWOM influence in case of B\&B industry. Prayukti - Journal of Management Applications, Vol. 1, Issue 1, pp. 19-28.

*Corresponding author e-mail: saurabhsood78@gmail.com

\begin{abstract}
The goal of this paper is to study the impact of the perceived diagnosticity on purchase intention and the mediating role of the perceived EWOM influence in case of $B \& B$ industry. 152 peer-reviewed journal articles were reviewed. Exploratory research design was utilized in this case. The major findings of the study were perceived diagnosticity had a strong impact on the purchase intention in case of B\&B industry. Also, perceived EWOM influence did have a mediating role to play between perceived diagnosticity and purchase intention. This research will help in improving the understanding of the practitioners about the perceived diagnosticity in case of online reviews. The practical significance of this study can be very significant and influential for the B\&B industry, as it would help business in understanding if providing a feature of posting reviews anonymously or with revealed identification has any impact on purchase intention. In case of academics, this research aims on filling the gap found in the literature, in relation to EWOM and B\&B industry. It is the first paper to study the effect of perceived diagnosticity in case of online reviews on the purchasing intention and to check the mediating impact of the perceived EWOM influence in $B \& B$ industry.
\end{abstract}

KEYWORDS: Bed and breakfast, Perceived source credibility, Perceived ewom influence, Source expertise, Purchase intention.

\section{INTRODUCTION}

Word of mouth scholars study both WOM culture and electronic WOM; such as researching the effects of EWOM on customer service/product choices (Bone, 1995; Engel et al., 1969; Feldman and Lynch, 1988) and EWOM's effect on plans to buy a product/service (Bone, 1995; Engel et al., 1969; Feldman and Lynch, 1988) (Brown and Reingen, 1987; Herr 
et al., 1991). When it comes to the sourcing of new services/products, customers have been known to rely much of the time on word of mouth (Arndt, 1967; Richins, 1983), one reason why people trust EWOM to gather data directly when they are in the shopping process is that EWOM really has no selling intent as in the ads (Schlosser, 2011; Sen and Lerman, 2007). Previous and existing tests have demonstrated that EWOMs have a very high control potential relative to traditional ads (Engel et al., 1969; Trusov et al., 2009).

EWOM has undeniably emerged as a very influential marketing force, and this is the main reason why EWOM research studies study different aspects of it (Chevalier, 2006). Studies linked to the implications of EWOM are seen to be largely distributed, so it has become harder to draw meaningful conclusions from them. Furthermore, researchers have used many analysis approaches to study the implications of the EWOM phenomenon, and none has been conducted to study its influence on the B\&B market. Websites listing B\&B providers should clearly describe the property in detail when promoting their businesses, along with the experiences to be provided. This is especially important for intangible facilities so visitors do not know what to expect (Kline et al., 2004). For the first time, it becomes important to do the right in consumer relations when B\&B owners may not get another opportunity to satisfy their clients (Kaufman and Weaver, 1998). This clearly illustrates that online reviews are of high importance for the B\&B market.

Online reviews are gaining value not only in the retail industry but also in the tourism industry, as there is a high possibility that when it comes to making an online reservation for a small property, before coming to the property website, the traveler will read the online reviews on other websites, Gretzel (2006). The Internet is used as a trip planning tool for today's travelers. According to Forrester Research (2002), over 64 million individuals have searched for travel information online or ordered travel goods online, with total sales of $\$ 20.4$ billion.

Research on the service is predictably very limited considering the recent introduction of $\mathrm{B} \& \mathrm{~B}$, although researchers in tourism and other fields are rapidly gaining interest. Many of the research available today was possibly published during the reporting period. A significant portion of Air B\&B surveys have been performed by various industry bodies, rather than academics. These apparent shifts in the current world have necessitated the need for studies in this area to explain these new episodes in order to build on these trends and to improve and adapt applicable digital marketing strategies accordingly.

India is on the way to becoming one of $B \& B$ 's biggest revenue drivers, as more than one million Indians have flown worldwide on B\&B. In about 10 years, India is projected to be one of the world's biggest B\&B markets, with an estimated demand of 230 million urban Indian millennials looking for unique and exciting ways to travel (Economic Times, 2017). According to the data shared by the UN and the IMF, India and China are the main players leading the major economies in the growth of GDP in 2018. By looking specifically at B\&B, a steady increase can be seen, i.e. 52 percent growth in the number of listings (Indian Brand and Equity Foundation, 2018).

Many existing and past studies suggest that the perceived dignity of an EWOM is an integral component of the actions of a customer (Cheung \& Thadani, 2012). Lis (2013) noted that the view of a customer of the credibility of an EWOM is that the use of information would have a beneficial impact on the final buying intent. The way a client forms the reputation of EWOM is an important topic for understanding the complete process of using feedback when comparing the different aspects of attitude related to buying.

The variables that influence the diagnosticity of EWOM, such as the accuracy of the argument, the amount of analysis and the validity are tested in some tests; however, the results are unclear and highly inconsistent, may be due to the different measurement methods (Hennig-Thurau, et al., 2004). When it comes to the final buying decision, the quality of review or EWOM shared online has an important part to play. In the case with both internet stores and online rating sites like Amazon, customers or users of a review are provided with a special feature, where they can decide whether they deem the review to be helpful. The academics drew the academics to the EWOM integrity problem and its helpfulness, but the 
judgment on the variables evaluating the legitimacy of EWOM remains uncertain (McKnight \& Kacmar, 2007; Lis, 2013).

\section{REVIEW OF LITERATURE}

It is said that information exchanged online or offline is of high quality only when it is complete, which ensures that the information shared is adequate in scope and width and sufficient for end users to comprehend a mission (Wang and Strong 1996). A customer interested in booking a B\&B service can only consider the ratings to be complete until he/she provides detailed details that is eventually beneficial in making the final booking. A detailed description of the lodge, the place on which it is designed, costs, staff friendliness and more are included in an exhaustive analysis.

The specifics of the information available by social networks are very limited in the case of the conventional WOM; and thus, it is very difficult for families and friends to access full information about any product or service, particularly when it comes to choosing an accommodation while on holiday. In the case of e-WOM, a totally different situation may be found where travelers have the possibility of accessing hotel or B\&B details from several online outlets (e.g. mini-bar pricing, condition of breakfast, state of furniture, friendliness of staff), which can aid them in their decision-making phase. Another point here is that if the information presented on the online sites is complete and of good quality, then the likelihood of this information being followed by travelers also improve. The arguments are important in high quality reviews because they have a powerful ability to compel a person to take a specific action (Bhattacherjee and Sanford, 2006, p. 811). In plain terms, it can be said that a quality review is the one that can convince its readers to take action and even often defend firms (Cheung et al., 2009). Many studies have previously been performed where it has been concluded that a high-quality analysis can affect a reader's attitude (Cacioppo et al., 1983; Sia et al., 1999).

Only where the information presented in the review has the potential to affect readers is an online review said to be of high quality (Bhattacherjee and Sanford 2005). There are several factors that are taken into account in the consistency of the EWOM, such as the message's importance, knowledge precision, comprehensiveness and timeliness (Cheung and Thadani 2010). If the readers don't grasp details easily, it also causes morale concerns, which they mention in the comment section (Ratchford 2001). Lin et al. (2013), stated in their analysis that online reviews that are not partial and readily understandable, so it is highly likely that the reader will have a favorable effect on the decision to buy. In their research, Kempf and Smith (1998) clarified that perceived diagnosis is said to be high when the duration and reach of online examination is greater. Most of the details presented in the content of the reviews or EWOM is text, so the discrepancies in the reviews play a critical role in presumed diagnosticity. In their research, Wang (2011) concluded that in the case of items such as shampoo, an emotional analysis is not very influential compared to a rational EWOM. A quality evaluation is also said to have a favorable effect on the perceived diagnosis.

\section{H1: Quality of a review positively impacts the perceived diagnosticity in case of $B \& B$ services.}

The presumed diagnosis of an online review can be defined as the degree and overall severity to which the review encourages a reader to make an educated purchasing decision. The interpretation of diagnostic tests by readers can be related to the scope of the study. Interesting perspectives have been generated by scholarly research on the ways in which this can affect the assumed diagnosis. In particular, online reviews, which are lengthy and accompanied by clear and rational explanations, have the ability to encourage confidence and, thus, the review can be said to be diagnostic (Metzger, 2007). Similarly, readers often don't want to read excessively comprehensive web reviews (Otterbacher, 2009). Reviews that are quick to read are seen as more diagnostic (Korfiatis, et.al, 2012). In addition, reviews that lack sufficient product/service knowledge may often be seen as a lack of reviewers' 
experience in the matter, therefore not finding them to be diagnostic (Ghose, 2011). In general, the greater depth of the feedback is shown to increase the perceived importance of a review to promote a smooth decision-making process (Metzger, 2007).

Diagnosticity was discussed in prior studies in conjunction with the message or material shared by EWOM (e.g. Lopez \& Sicilia, 2014). There have also been a few studies that have been able to relate the presence of EWOM (positive/negative) with its effect on consumer attitudes in other sectors (Yoon, 2007). In addition to these results, a few other experiments in different industries have also been undertaken that have been able to create a correlation between positive advertisements and the decision to purchase and sell the finished product (Lopez \& Sicilia, 2014). Mishra et al. (1993), noted that if an individual does not make sense of the information shared, then a decision-simplifying model is more likely to be chosen. Contrary to this, if all valid facts, high source credibility, high star ratings and a greater number of reviews are produced, then it is more likely that he/she will follow a transparent and systematic decision-making mechanism.

Chan and Ngai (2011) listed in their research the influence of electronic word of mouth on the final purchasing activity that has already been observed and discussed in many other literatures in different contexts (e.g. Chen et al., 2013). Steffes and Burgee (2008) stated in their study that the data collected from a digital/online medium is said to have more effect than word of mouth when it comes to final decision-making. Online advertisers influence their customers to read and write reviews of their purchased products or services and to share these reviews with prospective customers further, as reviews from people who have already purchased them. Park et al. (2007) claimed in his research that the volume and accuracy of EWOM and other aspects of it, such as meaning, diagnosticity, subjectivity, etc., are some of EWOM's very important features that play an important role in the processing of customer information. EWOM's effect on revenue is studied in numerous industries such as beer (Clemons et al., 2006), books on internet media (Chevalier \& Mayzlin, 2006), or films and sales (Liu, 2006), and the effect of all components of online analysis has shown varying effects in those tests. In the other hand, Clemons et al. (2006) discovered that valence has no effect on film revenue when Liu (2006) discovered that valence has an impact on beer revenues.

Past longitudinal studies (e.g., Bansal \& Voyer, 2000) prove the usefulness of the data source in EWOM's correspondence process. In the mentality of the receiver of the letter, the skill of the person who shares information has a vital role to play. This consequence holds valid in the case of tests also conducted in the case of WOM. The root of information that extends to the sender in the case of EWOM will affect the attitude of the receiver towards the shared EWOM review/message (Cheung et al., 2009).

In a study conducted in 2014 by Chua and Banerjee, it was hypothesized that a substantial, meaningful positive relationship existed between the scope of the feedback and the perceived diagnostic assessment. They stated that the significantly detailed online reviews were seen as more diagnostic than the shorter reviews. Another important finding of this research was that in the case of both quest and experience goods, a detailed analysis is considered to be a precedent for review diagnosticity, having a favorable effect on the mood of the respondent, thus also the final purchasing intention, thus establishing this hypothesis:

\section{H2: Perceived Diagnosticity positively impacts the purchase intention in bed and breakfast industry.}

A third explanatory mediator variable is added to investigate the casual correlation between the exogenous variable and the endogenous variable (Hair et al., 2013). The bootstrapping method in PLS-SEM is suitable for mediation research since bootstrapping does not entail any conclusions about the distribution of statistics for sampling and can be extended to small sample sizes (Hair et al., 2013). The first step is to evaluate the direct effect (i.e. p. 13) of the exogenous variable on the endogenous variable, which may be relevant if the mediator is not included, in order to perform a mediation study in PLS-SEM (Lynch \& Chen, Zhao, 2010). The next step is to include the mediator variable in the PLS path model if 
the direct path is significant, and to evaluate the importance of the indirect path (i.e. p12* p23).

The relevance of each direction p12 and p23 for this state is a sufficient prerequisite. After bootstrapping, the indirect path can be measured and, if the indirect effect is considered to be important, any of the direct Mediating Variable Independent Variable direction may be absorbed by the mediator.

\section{H3: Perceived EWOM influence has a mediating role to play between perceived diagnosticity and purchase intention.}

- H3a: Perceived Source Credibility have a significant impact on predicting the perceived EWOM influence in the case of B\&B industry.

- $\mathbf{H}_{3 \mathbf{b}}$ : Perceived EWOM influence have a significant impact on predicting the purchase intention in the case of $B \& B$ industry.

\section{RELIABILITY AND VALIDITY}

\begin{tabular}{|c|c|c|c|}
\hline \multicolumn{5}{|c|}{ Table:1. Reliability and Validity } \\
\hline Variables & $\begin{array}{c}\text { Cronbach's } \\
\text { Alpha }\end{array}$ & Composite Reliability & $\begin{array}{c}\text { Average Variance } \\
\text { Extracted (AVE) }\end{array}$ \\
\hline Perceived diagnosticity & 0.886 & 0.917 & 0.688 \\
\hline Purchase intention & 0.925 & 0.943 & 0.769 \\
\hline Perceived EWOM Influence & 0.775 & 0.848 & 0.534 \\
\hline
\end{tabular}

The CR values for all variables used in this analysis were found to be $>0.70$ (Hair et al., 2006) which provides for internal consistency). Table 1 also shows the AVE values for all latent variables used in this study. Such values were found to be more than the accepted value of 0.50 (Hair et al., 2006) and thus create a converging value of 0.50 (Hair et al., 2006) and therefore establish convergent validity. Thus, it can be said that the measurement model had a reasonable internal consistency and convergent validity.

\section{RESULTS}

\section{Descriptive Statistics}

407 responses were collected and analyzed in the software- PLS SEM. The descriptive analysis of the respondents are as follows:

A questionnaire was sent to 525 respondents, out of which the researcher received only 407 questionnaires. All these 407 people sent completed questionnaires and agreed that they had looked for online reviews before booking any B\&B lodge. 225 respondents out of 407 were females which constitutes to almost $55 \%$ of the total sample and 178 respondents were males which is $43 \%$ of the total sample. There were 4 people who preferred not to disclose their gender related information, in the questionnaire. 34\% of the sample belonged to the age group-31 -40 years old ie $34 \%$, followed by $27.5 \%$ of the respondents falling in the age group 41-50 years old. Respondents were from Delhi, Gurgaon, Bangalore, Pune and Mumbai, out of which almost 25\% of the respondents were in Mumbai and next 225 were from Gurgaon. $47 \%$ of the respondents were employed, followed by $19 \%$ of the people who were self-employed. $62 \%$ of the respondents who filled the questionnaire always looked for reviews before booking a $\mathrm{B} \& \mathrm{~B}$ lodge.

Inference: Figure: 1 . Shows that $52.9 \%$ of the variability in perceived diagnosticity was explained by Review Quality and $64.5 \%$ of the variability in purchase intention was explained by perceived Source credibility.

By conducting a blindfolding technique (Hair et al., 2013) with an omission distance of 7 the cross-validated redundancy $\mathrm{Q}^{2}$ values of all endogenous variables were calculated. For this analysis, perceived source credibility had a $\mathrm{Q}^{2}$ value of 0.318 ; and purchase intention had 0.491 , respectively. Since all $\mathrm{Q}^{2}$ values are $>0$, it is known that the structural model of the PLS has predictive relevance. 
$H_{1}$ : Quality of a review positively impacts the perceived diagnosticity in case of $B \& B$ services.

Figure 1: Impact of Review Quality on Perceived Diagnosticity and Purchase Intention

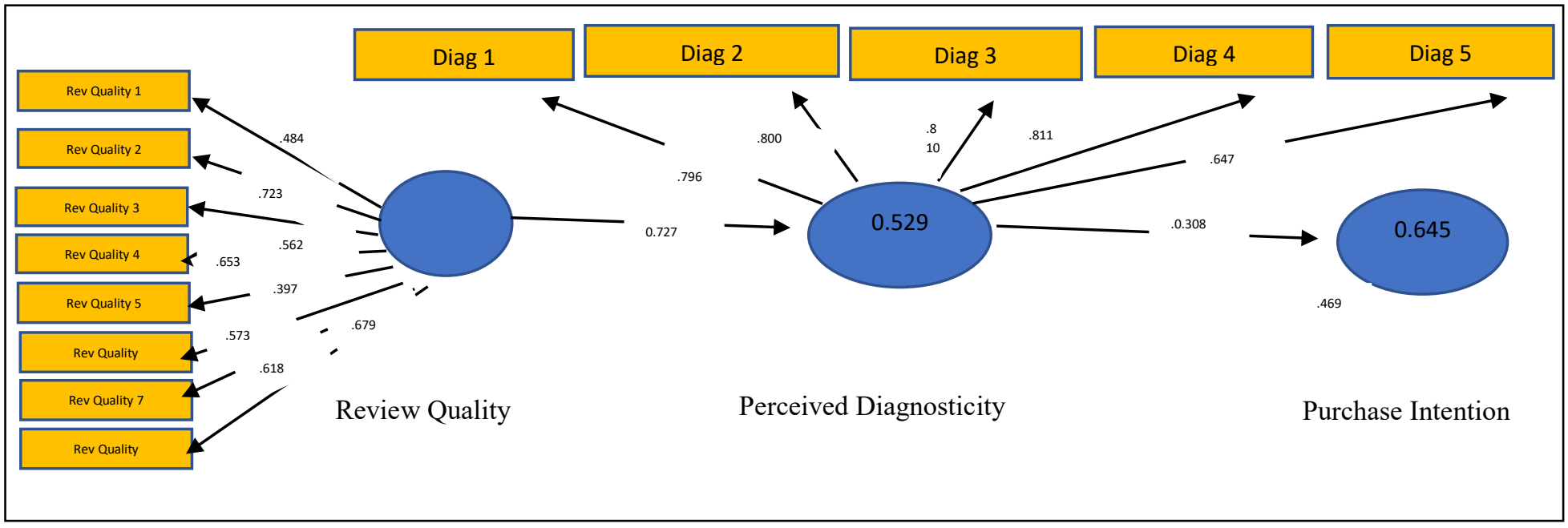

Source: Author's analysis

F2 size effect is the calculation used to determine the shift in the R2 value when the specified exogenous variable is omitted from the model (Hair Jr et al., 2013).

Small: $0.0<f 2$ effect size $<0.15$; Medium: $0.15<f 2$ effect size $<0.35$; Large: $f 2$ effect size $>0.35$.

\begin{tabular}{|c|c|c|}
\hline \multicolumn{3}{|c|}{ Table:2. F2 Values } \\
\hline & F2 & Status \\
\hline Perceived diagnosticity-->Purchase & 0.098 & Small \\
\hline Review Quality --> Perceived Diagnosticity & 0.90 & Large \\
\hline
\end{tabular}

The next step of the analysis was to analyse the relationship between the independent and dependent variables. This analysis used bootstrapping to check the Confidence intervals for path coefficients and statistical inference (Tenehaus et al., 2005). Based on the findings recorded in Table 3, the author concluded that all relationships which exist are statistically significant, as a result, the relationships proposed in $\mathrm{H}_{1}$ to $\mathrm{H}_{2}$ were optimistic and the pathways were significant.

\begin{tabular}{|c|c|c|c|c|c|}
\hline \multicolumn{2}{|c|}{ Table: 3. Path Coefficients (a) } \\
\hline \multicolumn{2}{|c|}{ Hypothesis } & $\begin{array}{c}\text { Path } \\
\text { Coefficients }\end{array}$ & t-value & P & Decision \\
\hline H1 & Review Quality--> Perceived Diagnosticity & 0.728 & 21.501 & 0 & Fail to reject \\
\hline H2 & Perceived Diagnosticity --> Purchase intention & 0.567 & 6.90 & 0 & Fail to reject \\
\hline
\end{tabular}

H3. Perceived EWOM influence has a mediating role between perceived diagnosticity and purchase intention, in case of B\&B industry.

$\mathrm{H}_{3 \mathrm{a}}$. Perceived Source Credibility have a significant impact on predicting the perceived EWOM influence in the case of B\&B industry.

$\mathrm{H}_{3 \mathrm{~b}}$. Perceived EWOM influence have a significant impact on predicting the purchase intention in the case of B\&B industry.

\section{a). Direct impact of independent variable should be significant}

$\mathrm{H}_{2 \mathrm{a}}$. Perceived diagnosticity have a significant impact on predicting the purchase intention in the case of $\mathrm{B} \& \mathrm{~B}$ industry. 


\begin{tabular}{|l|c|c|c|}
\hline \multicolumn{4}{|c|}{ Table: 4. Direct impact of independent variable is significant } \\
\hline & T Statistics $(|\mathrm{O} / \mathrm{STDEV}|)$ & P Values & Result \\
\hline Perceived diagnosticity $->$ Purchase intention & 6.90 & 0.00 & Significant \\
\hline
\end{tabular}

As seen in figure $1,64.5 \%$ of the variability in purchase intention was explained by perceived diagnosticity and path coefficient test confirmed that the relationship which exist between perceived diagnosticity and purchase intention are statistically significant

b). Indirect path where independent factors have an impact on perceived EWOM influence should be significant

$\mathrm{H}_{2 \mathrm{~b}}$. Perceived diagnosticity have a significant impact on predicting the perceived EWOM influence in the case of B\&B industry.

Figure:2. Impact of Perceived Source credibility and perceived EWOM Influence

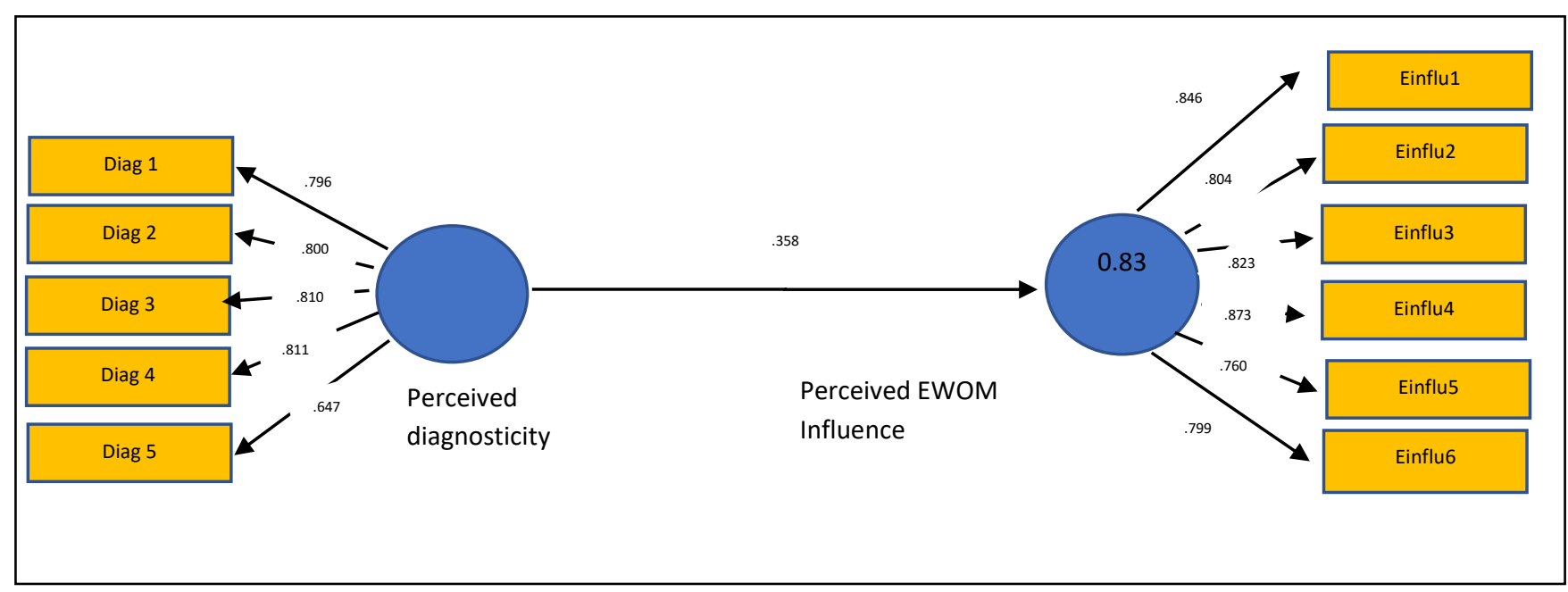

$83 \%$ of the variability in perceived EWOM Influence was explained by perceived diagnosticity and path coefficient test confirmed that the relationship which exist between perceived diagnosticity and purchase intention are statistically significant.

\begin{tabular}{|l|c|c|c|}
\hline \multicolumn{4}{|c|}{ Table:5. Indirect path } \\
\hline & $\begin{array}{c}\text { T Statistics } \\
(\mid \mathrm{O} / \text { STDEV } \mid)\end{array}$ & P Values & Result \\
\hline Perceived Diagnosticity -> Perceived EWOM influence & 8.97 & 0 & Significant \\
\hline
\end{tabular}

Table: 5 shows that the indirect path where independent factors have an impact on per EWOM influence is significant

\section{c). Impact of perceived EWOM influence on purchase intention is significant}

$\mathrm{H}_{2 \mathrm{c}}$. Perceived EWOM influence have a significant impact on predicting the purchase intention in the case of $\mathrm{B} \& \mathrm{~B}$ industry.

\begin{tabular}{|l|c|c|c|}
\hline \multicolumn{4}{|c|}{ Table: 6. Impact of perceived EWOM influence on purchase intention is significant } \\
\hline & $\begin{array}{c}\text { T Statistics } \\
(|\mathrm{O} / \mathrm{STDEV}|)\end{array}$ & P Values & Result \\
\hline Perceived EWOM influence -> Purchase intention & 61.359 & 0 & Significant \\
\hline
\end{tabular}

d). The final step here is to understand, how much of the direct path is absorbed, for which VAF is calculated:

$(\mathrm{p} 12 * \mathrm{p} 23) /(\mathrm{p} 13+\mathrm{p} 12 * \mathrm{p} 23)$. Based on the value of VAF, the following conditions of mediation effect are given by Hair et al. (2013, p.224): I If $0<\mathrm{VAF}<0.20$, then No 
mediation. ii) If $0.20<\mathrm{VAF}<0.80$, then part mediation. (iii) If $\mathrm{VAF}>0.80$, then Complete Mediation.

a). Mediation analysis: Per EWOM influence as a mediator

b). Mediation analysis: Per EWOM influence as a mediator

\begin{tabular}{|l|l|l|l|}
\hline \multicolumn{3}{|c|}{ Table: 7. VAF(d) } \\
\hline Independent variable & Dependent variable & VAF range & Mediation \\
\hline Perceived Diagnosticity & Purchase intention & 0.99 & Full \\
\hline
\end{tabular}

Figure: 3. Mediation analysis: Per EWOM influence as a mediator

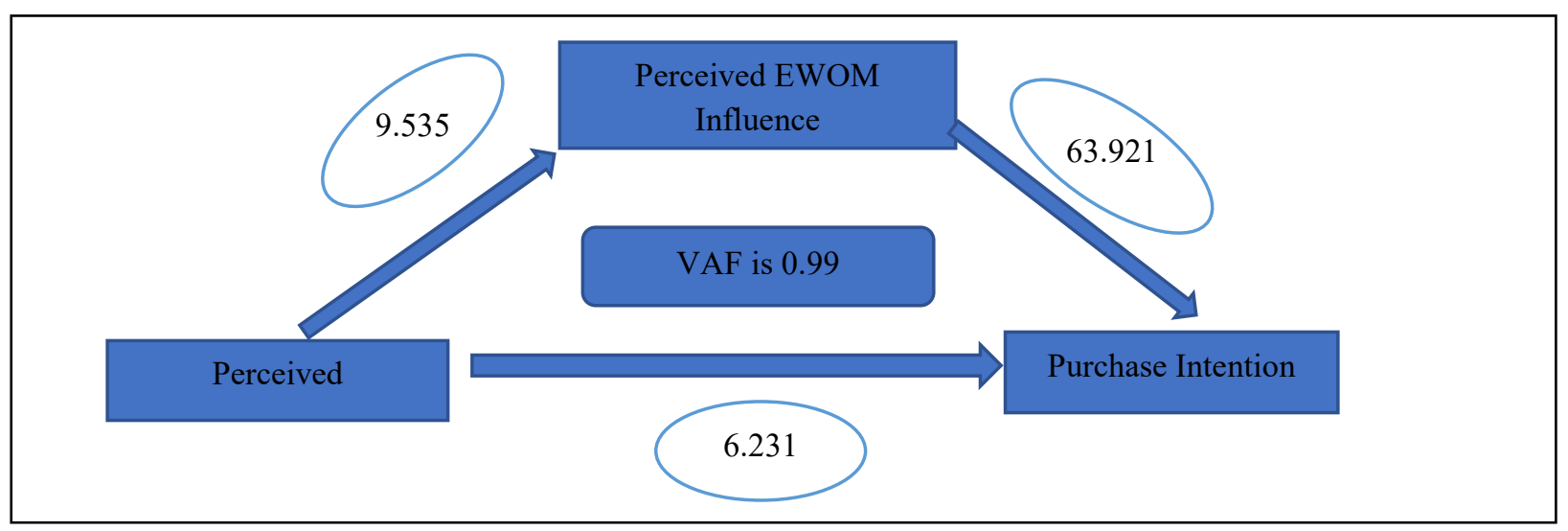

\section{DISCUSSION AND CONCLUSION}

First, figuring out whether the source expertise had a good connection which had a significant effect on the perceived diagnosticity, which was supported by several previous studies. There are a few researchers who have argued that if an person is an expert in some particular category of the product, he / she will be able to share more product / purchase related information, and as they have more expertise on the subject, their opinion would be taken care of more often than the others(Gilly et al. 1998). The findings that there is medium strength between review quality and perceived diagnosticity, is in confirmation with the studies conducted in the past with the fundamental basis that people will trust information which is diagnostic. In general, the greater depth of the feedback is shown to increase the perceived importance of a review to promote a smooth decision-making process (Metzger, 2007). In a study conducted in 2014 by Chua and Banerjee, it was hypothesized that a substantial, meaningful positive relationship existed between the scope of the feedback and the perceived diagnostic assessment. They stated that the significantly detailed online reviews were more diagnostic than the shorter reviews. Another important observation of this study was that a thorough review is considered to be a precedent for diagnostic review in the case of both search and experience products, creating a positive impact on the attitude of the respondent, thus also the final purchase intention Perceived EWOM Influence is the degree to which a respondent attitude has been influenced towards the EWOM by information accumulated from online reviews Mishra et al. (1993) and from the literature review it could be confirmed that it has an impact on the purchase intention. Here the researcher wanted to check if this holds true in case of B\&B industry too and here are the findings: The VAF value is high ie 0.99 confirming that perceived EWOM influence has a mediating role to play between perceived diagnosticity and purchase intention.

\section{Implication, limitation and Future scope}

This study is important for the marketing managers in understanding an important component of EWOM ie review quality and its impact on the consumer perception ie perceived diagnosticity. This will also help the managers in including these components in their website, like making sure that the consumers are given the option of sharing their star ratings besides the text review. Source Expertise could be checked by providing them 
different badges based on the type of information they share like expert, novice etc. This also fills a gap in the academic literature, in the case of B\&B services.

$\mathrm{B} \& \mathrm{~B}$ is in an initial stage in India, and therefore this study can shed more light on the feature that the entrepreneurs can put in their website, which will further give a sound information to the prospect customers. This research can also help customers in understanding how they can build their profile which might be helpful to build trust of the readers on the reviewers. This can further help them gain an expert reputation based on the badges that are defined by the company.

Here a limitation could be that the researchers should include other components of EWOM too, like the quality of the reviews, number of reviews and check its impact on the perceived diagnosticity \& valence. Next limitation could be that, only some metropolitans in India were selected as a location for study, here it is suggested to conduct similar studies in other regions to check if the similar observation were seen there too. In this study the focus was only on major five metropolitans and therefore more researches should be done in other Tier- 2 and tier-3 cities. It is suggested that this study should be replicated in other geographies and in developing economies specifically, to check if similar results were observed. Additionally, researchers can deep dive into other EWOM components and check its impact on the perception of consumers in B\&B industry. This study could also be replicated in other industries too. The sampling method used here was of convenience sampling which is subjected to biasness and therefore a similar study with random sampling method should be conducted. Researchers can include other variables and check the moderating and mediating impact on the final purchase intentions.

\section{REFERENCES}

- Arndt, J. (1967). Role of product-related conversations in the diffusion of a new product. Journal of Marketing Research, 4, 291-295.

- Bansal, H.S. and Voyer, P.A. (2000). Word-of-mouth processes within a service purchase decision context. Journal of Service Research, Vol. 3 No. 2, pp. 166-77.

- Beltramini, R. F. \& Sirsi, A. K. (1992). Physician information acquisition and believability. Journal of Health Care Marketing, 12(4), pp. 52-59.

- Bhattacherjee, A., \& Sanford, C. (2006). Influence Processes for Information Technology Acceptance: An Elaboration Likelihood Model. MIS Quarterly, 30(4), 805-825. doi: $10.2307 / 25148755$

- Bickart, B. Schindler, R. (2001). Internet forum as influential sources of consumer information. Journal of Interactive Marketing, 1(3), 31-40.

- Bloch, Peter H. Richins, M. (1983). A Theoretical Model for the Study of Product Importance Perceptions. Journal of Marketing, 47 (Summer), 69-81.

- Bone, P. F. (1995). Word-of-mouth effects on short-term and long-term product judgments. Journal of Business Research, 32, 213-223.

- Bristor, Julia M. (1990). Enhanced Explanations of Word-of-Mouth Communication: The Power of Relationships. Research in Consumer Behavior, 4 (1), 51-83

- Brown, J.J. Reingen, P.H. (1987). Social ties and word-of-mouth referral behavior. Journal of Consumer Research, 14(3), 350-362.

- Chan, Y.Y.Y. \& Ngai, E.W.T. (2011). Conceptualizing electronic word of mouth activity: An input-process-output perspective. Marketing Intelligence \& Planning, 29(5), 488-516.

- Chen, C. Lurie, N. H. (2013). Temporal contiguity and negativity bias in the impact of online word-of-mouth. Journal of Marketing Research, 50(4), 463-476.

- Cheung C.M.K., Lee M.K.O., Thadani D.R. (2009). The Impact of Positive Electronic Word-of-Mouth on Consumer Online Purchasing Decision. In: Lytras M.D. et al. (eds) Visioning and Engineering the Knowledge Society. A Web Science Perspective. WSKS. Lecture Notes in Computer Science, vol 5736. Springer, Berlin, Heidelberg. 
- Cheung, C.M. Thadani, D.R. (2012). The impact of electronic word-of-mouth communication: A literature analysis and integrative model. Decision Support Systems, Vol. 54 No. 1, pp. 461-470.

- Chevalier, J. Mayzlin, D. (2006). The effect of word of mouth on sales: Online book reviews. Journal of Marketing Research, 43(3), 345-354.

- Clemons, E.K. Gao, G.G. Hitt, L.M. (2006). When online reviews meet hyper differentiation: A study of the craft beer industry. Journal of Management Information Systems, 23(2), 149-171.

- De Bruyn, A. Lilien, G.L. (2004). A multi-stage model of word-of-mouth through electronic referrals. eBusiness Research Center, February.

- Engel, J. Roger, E. Blackwell, D. Robert, J. K. (1969). How information is used to adopt an innovation. $J$ Advert Res, 9 (3).

- Feick, L. and Higie, R.A. (1992), "The effects of preference heterogeneity and source characterisitcs on ad processing and judgments about endorsers", Journal of Advertising, Vol. 21 No. 2, pp. 9-24.

- Feldman, J. and Lynch, J.G. (1988). Self-generated validity and other effects of measurement on belief, attitude, intention and behavior. Journal of Applied Psychology, Vol. 73, August, pp. 421-35.

- Flanagin, A. J., \& Metzger, M. J. (2000). Perceptions of Internet information credibility. Journalism \& Mass Communication Quarterly, 77(3), 515-540.

- Froster, in: http://www.pageaccess.com/whitepapers/500ForresterUSInteractiveMktgForecast_2009_2014.pdf. [Accessed 20 June 2018].

- Kumar, A.; Gawande, A. and Brar, V. (2020). Neuro-Marketing: Opportunities and Challenges in India. Vidyabharati International Interdisciplinary Research Journal, Vol. 10, Issue 02, pp. 214-217. 